\title{
Conduction Disturbances after the Superior Septal Approach for Mitral Valve Repair
}

\author{
Yoshio Misawa, Shin-ichi Ohki, Yuichiro Kaminishi, Yasuhito Sakano, \\ Kei Aizawa, Tsutomu Saito, Hiroaki Konishi and Arata Muraoka \\ Division of Cardiovascular Surgery, \\ Jichi Medical University, \\ Japan
}

\section{Introduction}

The better the operative field, the safer the procedure. In mitral valve surgery, the approach to the left atrium influences the quality of the operation, especially in difficult anatomical situations such as a small left atrium, or technically difficult operations including mitral valvuloplasty. However, it is not always easy to get a good operative field for mitral valve surgery, particularly in cases with a small left atrium.

The conventional approach includes the left atriotomy from the right side and the so-called septal approach. These do not sacrifice the sinus node artery. In contrast, the superior septal approach sacrifices the sinus node artery, and it requires a more invasive incision into the right and left atria. Therefore, postoperative rhythm disturbances, such as supraventricular arrhythmia and atrio-ventricular conduction changes, could arise in patients who had mitral valve surgery via the superior septal approach. However, the superior septal approach provides surgeons with a large operative field even when the left atrium is small. With the superior septal approach, the surgeon and assistants can clearly observe the operative field, whereas with the conventional approach the operative field is not always fully visualzed.

Some studies of rhythm disturbances accompanying the superior septal approach have been published previously, but they covered mainly perioperative events, such as premature contraction and bradycardia. We evaluated perioperative events and postoperative cardiac conduction disturbances in patients who had mitral valve operations by the superior septal approach and compared them with patients who had the conventional surgical approach.

\section{Patients and methods}

\section{a. Patients profiles:}

Between October in 1996 and October in 1998, 52 patients (25 male, 27 female) had mitral valve operations by the superior septal approach, and cardiac rhythm status was assessed (Misawa et al., 1999). The average age of the 52 patients was $55+/-14$ years (range, 15-76 years). Preoperative mean pulmonary artery pressure was $29+/-11 \mathrm{mmHg}$, and the mean left atrial diameter was $63+/-10 \mathrm{~mm}$.

Twelve patients ( 7 male, 5 female) who underwent mitral surgery by the conventional left atriotomy from the right side served as the control group. The average age of the group was 
$59+/-10$ years (range, 36-72 years). Preoperative mean pulmonary artery pressure was 33 $+/-8 \mathrm{mmHg}$, and the mean left atrial diameter was $68+/-19 \mathrm{~mm}$.

b. Our superior septal approach:

To establish cardiopulmonary bypass, bicaval venous drainage is essential for the superior septal approach, and direct caval cannulation is used for the drainage from the superior vena cava. The myocardium was protected during operation with moderate hypothermia (28-32 degree Celsius) and intermittent ante-grade infusion of blood cardioplegic solution. In the case of bradycardia below 70 beats/min, right atrial or ventricular pacing between 70 and 90 beats/min was initiated to obtain a stable postoperative hemodynamic condition.

Our procedure includes a right atriotomy not going beyond the crista terminalis, an atrial septotomy beginning at the fossa ovalis, and a subsequent left atrial incision parallel to the superior vena cava (Figure 1). We did not extend the incision onto the superior portion of the left atrium behind the aorta, but made it parallel to the superior vena cava. The incision length is limited up to a maximum of $2 \mathrm{~cm}$.

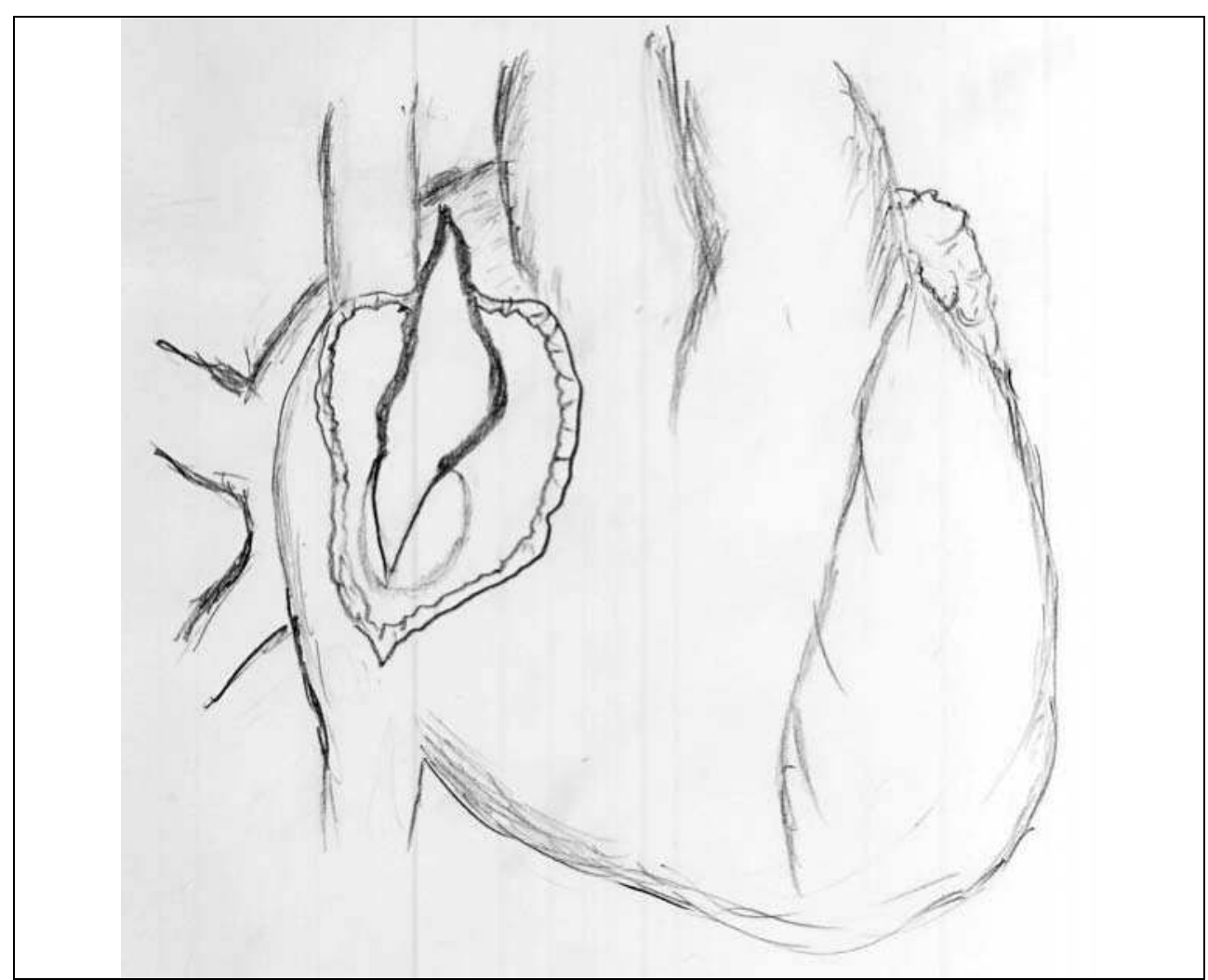

Our procedure includes a right atriotomy not going beyond the crista terminalis, an atrial septotomy beginning at the fossa ovalis, and a subsequent left atrial incision parallel to the superior vena cava. We did not extend the incision onto the superior portion of the left atrium behind the aorta, but made it parallel to the superior vena cava. The incision length is limited up to a maximum of $2 \mathrm{~cm}$.

Fig. 1. Our procedure of the superior septal approach 


\section{c. Operations:}

Forty-two patients of the superior septal approach group underwent mitral valve replacement, and 10 had mitral valvuloplasty. Twenty-two patients had concomitant operations, including aortic valve replacement in nine, tricuspid annuloplasty in 12, coronary arter bypass grafting in one, atrial septal defect repair in one, atrial septal defect repair plus tricuspid annuloplasty in one, and patch plasty for abscess cavity in the mitral annulus in one. Five patients had previous mitral or aortic valve operations. Cardiopulmonary bypass time was $158+/-22$ minutes, and ischemic time was $106+/-18$ minutes.

Eleven patients of the conventional approach group underwent mitral valve replacement, and one had mitral valvuloplasty combined with mitral valve annuloplasty. Three patients had tricuspid annuloplasty, three had the aortic valve replaced, and four had coronary artery bypass grafting. Cardiopulmonary bypass time was $172+/-45$ minutes, and myocardial ischemic time was $111+/-31$ minutes.

\section{d. Postoperative follow-ups and complications:}

The mean follow-up period of the superior septal approach group was $30+/-7$ months (range, 23-45 months). The mean follow-up period of the conventional approach group was $15+/-8$ months (range, 2-25 months).

Postoperative complications were compared in the two groups, and pre- and postoperative electrocardiograms were analyzed in patients with normal sinus rhythms. Serial changes in PR intervals were assessed to study the conduction disturbances that occurred with the two approaches. Holter electrocardiograms were used 6 to 12 months after surgery to assess cardiac rhythm status in the superior septal approach group.

Postoperative mortality and morbidity was analyzed upon the revised guideline (Edmunds et al., 1996). Statistical analysis was based on computer software "STAX '98" produced by Nakayama-shoten, Tokyo, 1998. Data are presented as mean $+/$ - standard deviation, and $p$ values less than 0.05 were considered significant.

\section{e. Additional studies}

Results from a further 61 superior septal approach patients were also included giving a total of 113 patients (Misawa \& Kaminishi, 2004). The superior septal approach is also suitable for a left atrial tumor. Recently, the tumor is incidentally diagnosed by echocardiography or chest computed tomography (Misawa et al, 2002).

\section{Results}

\section{a. Postoperative complications:}

In the 52 patients with the superior septal approach, there was no operative death. No intractable arrhythmias occurred in the early postoperative phase. There was one late death from cerebral hemorrhage 4 months postoperatively. Paravalvular leakage was found in one patient 2 months postoperatively, and this patient had a second operation 14 months after the initial surgery because of advancing heart failure. One cirrhotic patient with both aortic and mitral valve replacement underwent reexploration because of mediastinal bleeding 4 hours postoperatively. There were no other valve-related or treatment-related complications.

In the control group of the 12 patients, no intractable arrhythmias occurred in the early postoperative phase. Low cardiac output syndrome developed in the patient who had mitral valvuloplasty 3 hours after the initial operation, and the patient subsequently underwent mitral valve replacement. No other complications were observed in the control group. 


\section{b. Changes of postoperative electrocardiograms:}

Twenty-six patients had normal sinus rhythms preoperatively. Eleven had transient atrial fibrillation or junctional rhythms for approximately 2 weeks. Twenty-five patients maintained sinus rhythm, and there was atrial fibrillation in one patient at the final followup. Five of 26 patients with preoperative atrial fibrillation showed sinus rhythm in the early postoperative phase. In one patient atrial fibrillation recurred within 6 months postoperatively, and another died from cerebral bleeding 4 months postoperatively. Another 10 patients with atrial fibrillation showed transient junctional rhythms postoperatively.

Serial changes in PR intervals were assessed in the 25 patients who maintained their sinus rhythms (Figure 2). The mean preoperative PR interval was 155 +/- 20 milliseconds, indicating no conduction delay. However, postoperative PR intervals were significantly prolonged for 1 week $(p=0.02)$, decreased within 2 weeks postoperatively, and returned to normal by 6 months postoperatively. Five patients developed transient first degree atrioventricular blocks, and one developed a permanent first degree atrioventricular block. No other conduction abnormalities were observed.

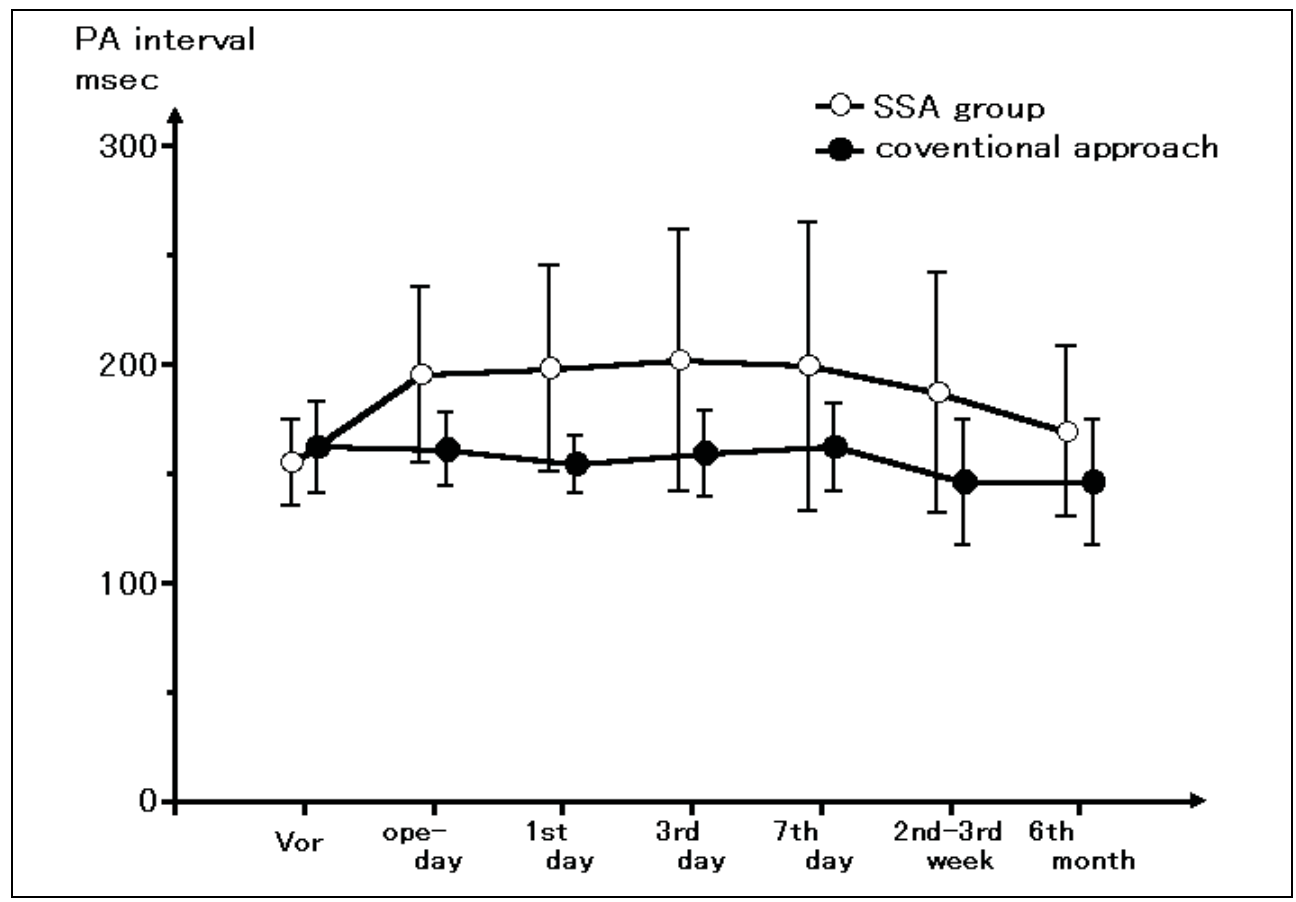

PR intervals of patients undergoing the superior septal approach become longer postoperatively and decreased gradually. Those of patients undergoing the conventional approach show no significant change significantly ( $p$-values between any PR intervals $>0.10$ ). Between the two groups, there is a significant difference in serial PR interval changes ( $p=0.05$ by the generalized Wilcoxon test).

Abbreviations: SSA; superior septal approach, ope-day; operation day, msec; milliseconds

Fig. 2. Postoperative serial PR interval changes 
Twenty-seven superior septal approach patients underwent Holter electrocardiograms more than 6 months postoperatively. No patient showed bradycardia below $50 / \mathrm{min}$. Supraventricular arrhythmias such as premature atrial contractions were limited within $3 \%$ of the total beats. Cardiac rhythms responded to the patients' activity levels. None of the 52 superior septal approach patients required pacemaker implantation.

Postoperative echocardiography 1-2 months after operation revealed no case of atrial septal defect.

Five patients maintained normal sinus rhythms perioperatively in the control group. The other patients remained atrial fibrillation throughout the follow-up periods. Mean PR interval of the five patients with sinus rhythm was 162 +/- 21 milliseconds preoperatively, with no significant change postoperatively ( $p$-values of any PR intervals are more than 0.10 , Figure 1). No patient developed atrioventricular blocks. There was a significant serial change between the two groups $(p=0.05)$. None of the 12 patients in the control group also required pacemaker.

c. Additional studies:

Including patients from the additional study, 64 of 66 patients with sinus rhythm preoperatively maintained sinus rhythm postoperatively, and seven of 47 with preoperative atrial fibrillation regained sinus rhythm at final follow-up. No patient needed pacemaker implantation, but two had a pacemaker implanted preoperatively.

A left atrial tumor is also a candidate for the superior septal approach (Misawa et al, 2002). We encountered a cardiac myxoma which located on the superior wall of the left atrium. The endoscope-assisted superior septal approach gave an excellent operative view and provided for a safe tumor resection. A 74 year-old man who had been experiencing paroxysmal atrial fibrillation for a few months was admitted to our hospital. Echocardiographic and computer tomographic studies showed a round, homogeneous mass at the free wall of the left atrium. The left atrium was echocardiographically measured at 39 $\mathrm{mm}$ in depth beneath the ascending aorta. The patient consented to resection of the tumor. Extracorporeal circulation was established through conventional median sternotomy, and moderate hypothermia and blood cardioplegic solution was used for myocardial protection. The right atrium and atrial septum were incised. A thoracoscope, which is used for routine endoscopic thoracic surgery in our institute, inserted through the atrial septotomy revealed that the stalk of the tumor was located on the superior wall of the left atrium. The incision was extended from the atrial septum to the superior aspect of the left atrium, without incising the stalk (Figure 3). Under thoracoscopic observation, the incision was completed along the superior wall of the left atrium. The stalk was excised together with an adjacent 5 $\mathrm{mm}$ of left atrial intima. The intimal defect was closed by direct suture without a patch. Myocardial ischemia time was 55 minutes. Pathological examination showed a completely resected $28 \times 25 \times 17 \mathrm{~mm}$ mass compatible with cardiac myxoma. He was discharged with no complications.

\section{Comment}

a. Cardiac surgery and postoperative conduction disturbances:

The risk of developing conduction disturbances after cardiac surgery has been well established. Goldman and associates retrospectively reviewed 5,942 patients who underwent open-heart surgery for acquired heart disease, and they revealed that 123 patients $(2.1 \%)$ required permanent cardiac pacing postoperatively; $4.6 \%$ of these underwent 


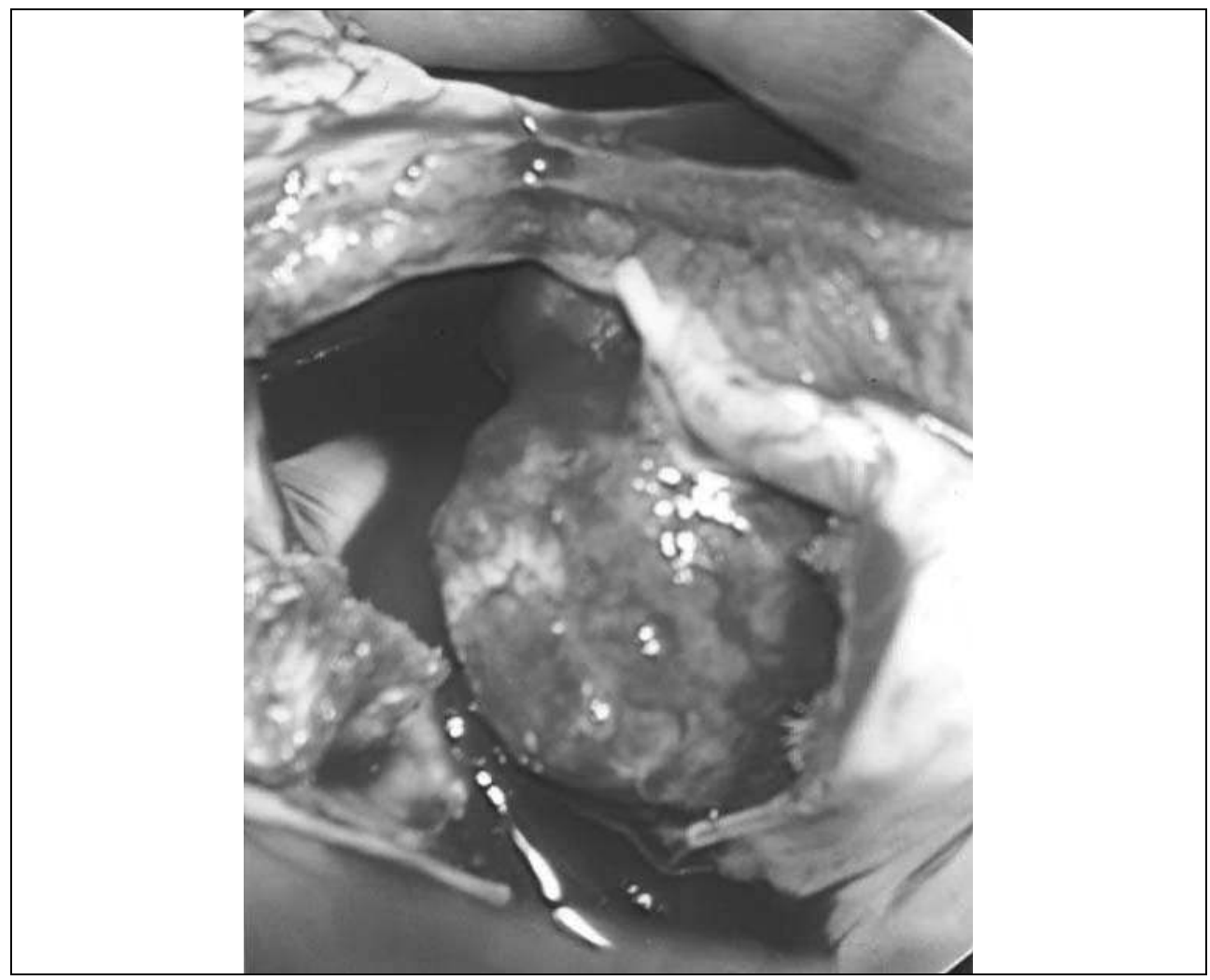

Myxoma of the left atrium was removed via the superior septal approach. The tumor originated from the free wall of the left atrium.

Fig. 3. An operative view in a case of a left atrial tumor

predominantly valvular surgery and $0.6 \%$ had coronary bypass (Goldman et al., 1984). They also showed that risk factors of postoperative pacemaker implantation appeared to be: 1) preoperative evidence of a conduction disorder; 2) advanced patient age; 3) dense calcium in the aortic annulus; 4) valvular surgery and, especially, tricuspid valve surgery; and 5) poor myocardial protection.

Another interesting article was published, referring to permanent pace maker implantation following cardiac surgery (Merin et al., 2009). They reviewed 4,999 patients undergoing surgery between 1993 and 2005. The average patient age was $64+/-12$ years, and $71 \%$ were males. Coronary bypass was performed in 4,071 , aortic valve replacement in 675 , and mitral valve replacement in 968 patients. Seventy-two patients $(1.4 \%)$ required pacemaker implantation after surgery. Indications for pacemaker implantation included complete atrioventricular block in 59, symptomatic bradycardia/slow atrial fibrillation in nine, second-degree atrioventricular block in two, and other conduction disturbances in two patients. Left bundle branch block and aortic valve replacement were predictors for pacemaker implantation. Of those receiving a pacemaker, about one-third will recover at late follow-up. They did not analyze the differernces in approach in mitral valve surgery, 
but their study implied that any cardiac surgery has a potential risk of postoperative pacemaker implantation.

Emkanjoo and associates revealed any types of conduction disturbances after open heart surgery (Emkanjoo, et al., 2008). This prospective study included coronary artery bypass $(n=128)$, valve surgery $(n=95)$, coronary artery and valvular surgery $(n=6)$, repair of ventricular septal defect $(n=51)$, repair of tetralogy of Fallot $(n=57)$, and others $(n=37)$. Among 374 patients, 192 developed new conduction disorders including symptomatic sinus bradycardia, atrial fibrillation, first to third atrioventricular block, and new right or left bundle branch block. In $5.6 \%$ patients, a permanent pacemaker was implanted, $47.6 \%$ of them underwent valvular surgery. In the coronary artery bypass group, $29.7 \%$ of patients developed new conduction disturbances; the most common of them was symptomatic sinus bradycardia. After valvular surgery $44.2 \%$ of patients developed conduction disturbances, the most common being atrial fibrillation with a slow ventricular response. After ventricular septal defect and tetralogy of Fallot repair, the most common conduction disturbance was new right bundle branch block. In $44.1 \%$ of patients the conduction defects occurred in the first 48 hours after surgery. The study showed that valvular surgery has a high potential risk of conduction disturbances during the early postoperative phase.

\section{b. Approaches to the mitral valve and a small left atrium:}

Conventionally, a left atriotomy made posterior to the interatrial groove or a transseptal approach was chosen for the mitral valve surgery. However, these approaches often resulted in a limited operative field in the case of a small left atrium or reoperation. Poor observation in the limited field might compromise surgical accuracy. Mitral valvuloplasty, which is more favored than valve replacement because of a better quality of life postoperatively, requires optimal exposure of the valve and the subvalvular apparatus. In such a case, precision and a secure reliable technique are necessary for successful results. Therefore, good exposure of the mitral apparatus is essential for the principal procedure. In addition, a young surgeon would also be able to perform the surgery successfully under a wide operative field where the instructor could also closely observe his procedure. The superior septal approach can provide a wider operative field than conventional approaches. A tumor can sometimes be found incidentally in the left atrium. In such a case, the left atrium is often small and the superior septal approach is suitable (Misawa et al., 2002). Our patient's small atrium and tumor site prevented us from observing the tumor stalk by the conventional transseptal view. With endoscopic examination, the anatomy of the tumor was clarified, and subsequent operative procedures were easily performed. Our thoracoscope was not flexible because it was designed for use in thoracic surgery. Use of a flexible endoscope in intracardiac procedures would be even more desiable in cases such as ours. With further innovation in endoscopic instruments, complex surgical procedures requiring subtle maneuvers will be possible under endoscopic assistance. Our case exemplifies the increasing usefulness of endoscope-assisted cardiac surgery.

\section{c. Mitral vlave surgery and postoperative conduction disturbances:}

Takeshita and associates reviewed the electrocardiograms of 76 patients who underwent mitral valve operations either via the transseptal superior approach or via the right lateral atriotomy (Takeshita et al., 1997). Nine patients who maintained the sinus rhythm for more than one year after surgery via the transseptal superior approach were selected for electrophysiological study to evaluate the sinus node function. They concluded that postoperative electrocardiographic and electrophysiological studies revealed that the sinus node function after the transseptal superior approach was relatively well maintained for 
more than one year after the operation. The influence of the transseptal superior approach on the sinus node function in the mid-term postoperative period was apparently mild and did not cause a serious problem, and that some of the patients did show abnormal data in terms of sino-atrial conduction time and intrinsic heart rate. Therefore, they recommennded that further follow-up of the sinus node function is necessary in patients who underwent mitral surgery through the transseptal superior approach.

Some investigators mentioned a high risk of loss of sinus rhythm with the superior septal approach, but others showed that the superior septal approach was not associated with a greater incidence of rhythm disturbances. Masuda and assiociates found a higher incidence of dyshythmias with the superior septal approach in the early postoperative period than that with the conventional right lateral left atriotomy and a similar incidence of them in the late period (Masuda et al., 1996). The authors analyzed 152 consecutive patients who underwent mitral valve procedures between 1992 and 1995. Follow-up ranged from 2 to 38 months, and the mean follow-up was 13.8 months in the superior septal group of 83 patients and 16.1 months in the conventional group of 69 patients. They showed that the mortality rate was similar in the two groups, and the causes of death were not related to the left atriotomy. At discharge, $78 \%$ of those in the superior septal group remained in sinus rhythm and $96 \%$ of the patients in the conventional group who were in sinus rhythm preoperatively. At the last follow-up, $83 \%$ of these patients in the former and $88 \%$ in the latter remained in sinus rhythm. They concluded that although the incidence of dysrhythmias was higher with the superior septal approach in the early postoperative period, the approach provides an excellent operative view of the mitral valve and similar results in terms of late postoperative cardiac rhythms in comparison with the right lateral left atriotomy.

Lukac and associates retrospectively analyzed consecutive 577 patients after mitral valve surgery with the superior septal approach or the left atrial approach (Lukac et al., 2007). They used the superior septal approach in 150 patients and the left atrial approach in 427. Forty-four patients had a pacemaker implanted after the surgery; 17 in the superior septal group and 27 in the left atrial group $(p=0.010)$. Nineteen patients had a pacemaker implanted because of sinus node dysfunction; 9 in the former and 10 in the latter $(p=0.017)$. They concluded that the superior septal approach has a higher risk of clinically significant sinus node dysfunction than the left atrial approach, and that the risk of pacemaker implantation because of atrioventricular conduction disturbances was not different between the two groups.

Utley and Leyland compared the preoperative status, operative factors, and postoperative outcomes among patients having mitral valve operations with three atrial incisions (Utley \& Leyland, 1995). The incisions were right lateral $(\mathrm{n}=66)$, superior septal $(\mathrm{n}=46)$, and transseptal $(n=37)$. Patients in the superior septal group more commonly required permanent pacemakers than those in the right lateral group. In patients with sinus rhythm before operation, sinus rhythm had returned before hospital discharge more commonly in those in the right lateral group (35 of 44, 80\%) than in those in the superior septal group (18 of $28,46 \%$ ) or in the transseptal group (9 of $13,69 \%$ ). With multiple regression analysis the type of atrial incision was not a predictor of postoperative pulmonary failure or need for permanent pacemaker implantation. Right lateral and transseptal atrial incisions were predictors of retention of sinus rhythm after operation. They concluded that the results of the superior septal incision were comparable with those of other incisions except for a slightly greater risk of loss of sinus rhythm, and that one must weigh the technical advantages of the superior septal incision against the risk of loss of sinus rhythm. 
Kon and associates used the extended transseptal approach to the mitral valve for 71 consecutive procedures (Kon et al., 1993). Four patients died; none had complications directly attributable to the exposure. Twenty underwent a primary reparative procedure; 30 , a primary replacement procedure; and 21, a repeat procedure. Despite division of the sinus node artery, 26 of 32 patients with sinus rhythm preoperatively had sinus rhythm postoperatively; 4 had atrial fibrillation postoperatively. Twenty-seven of 37 patients with atrial fibrillation preoperatively had atrial fibrillation postoperatively; 8 had sinus rhythm postoperatively. They use the extended transseptal approach routinely for mitral valve operations because the exposure provided by the extended transseptal approach is superior to that of standard approaches.

Gaudino and associates evaluated the safety and effectiveness of the superior septal approach for routine mitral valve replacement (Gaudino et al., 1997). One hundred forty-six consecutive patients undergoing mitral valve replacement at their institution were randomly assigned to undergo the procedure using either the conventional left atriotomy or the superior septal approach. Postoperatively and during the follow-up, 12-lead electrocardiography, 24-hour Holter monitoring, and transthoracic and transesophageal echocardiography were performed in all patients. They showed that the cardiopulmonary bypass and cross-clamp times were significantly higher in the superior septal group. No significant difference in blood loss was found between the two groups, and no residual atrial septal defect was found in the superior septal patients. The maintainance of sinus rhythm at late follow-up and the incidences of postoperative arrhythmias and newly developed atrioventricular block were not significantly different between the two groups. They concluded that the use of the superior septal approach to the mitral valve was not associated with a greater incidence of rhythm disturbances or other complications.

In addition, Smith showed an interesting change of $P$ wave axis and morphology in patients who had the superior septal approach (Smith, 1992). The author used the superior septal approach for an excellent exposure in seven patients in whom there were a variety of obstacles to a conventional approach. Four had complex reoperations. There were no bleeding complications. At late follow-up there was no change in rhythm or conduction in four patients with atrial fibrillation preoperatively. A change in $\mathrm{P}$ wave axis and morphology was seen at late follow-up in 2 patients with normal sinus rhythm preoperatively, possibly related to division of the sinus node artery. A third patients with normal sinus rhythm preoperatively remained in normal sinus rhythm at late follow-up. The author also confirmed that the superior septal approach could be useful in complex reoperations, in procedures requiring right atriotomy for other reasons, and in patients with a small or inaccessible left atrium.

Yamada and associates reported postoperative premature ventricular contractions arising from the mitral annulus (Yamada et al, 2009). They showed that electroanatomic mapping during the premature ventricular contractions revealed a centrifugal activation pattern arising from the mitral annulus, and the premature ventricular contractions were likely to be idiopathic. They successfully achieved radiofrequency ablation at the site close to the anteroparaseptal end of the mitral annuloplasty ring, which was located adjacent to the fibrous trigone. Their experience indicated that premature ventricular contractions after mitral valve surgery could occur in any approaches to the left atrium.

\section{d. Sinus node function after mitral valve surgery:}

Silva Junior and associates studied the interatrial conduction times and atrial node performance in patients submitted to mitral valve surgery with the aid of temporary atrial 
epicardic electrodes (Silvia et al, 2008). Their approach to the mitral valve was a conventional left atriotmy The atriograms were carried out in the first postoperative day and before the hospital discharge of ten consecutive patients. Sixty percent of the patients could complete the post-operative study protocol. The main results were: a) Post-operative arrhythmias were detected in $50 \%$ of the patients; b) There were no statistical differences between the pre and post-operative 12 lead electrocardiograms;. c) The interatrial conduction time ranged from 90 to 140 milliseconds in the first post-operative day, and from 110 to 130 milliseconds at hospital discharge; d) The sinus node recovery time ranged from 250 to 560 milliseconds in the first post-operative day and from 180 to 360 milliseconds at hospital discharge; e) The sinus atrial conduction time remained between 70 and 140 milliseconds, both in the first post-operative day and at hospital discharge, and; f) The interatrial conduction time was normal in patients whose left atrium was less than $50 \mathrm{~mm}$ in diameter but supranormal in the remaining cases. They concluded that sinus node function and interatrial conduction are not altered by mitral valve operation via the conventional leftsided approach. Their data indicated that the conventional approach to the mitral valve may be more preferable than the superior septal approach from the point of conduction disturbences.

e. Causes of postoperative rhythm disturbances after the superior septal approach:

The sinus node artery arises from the right coronary artery in about $55 \%$ of hearts and from the left circumflex or main coronary artery in the remainder. When it originates from the right coronary artery, it runs superiorly over the wall of the right atrium beneath the right appendage to the base of the superior vena cava (Kirklin \& Barratt-Boyes, 1993). The sinus node artery must be frequently sacrificed for the superior septal approach, causing potential risks for postoperative arrhythmias such as supraventricular arrhythmias and atrioventricular conduction disturbances. There will be collateral blood flows in the postoperative recovery phase, but blood supply will be reduced in the early postoperatrive phase. Moreover, an atrial incision required for the superior septal approach is longer than that for conventional approaches, which might interfere with the cardiac conduction system, resulting in conduction disturbances and arrhythmias.

Berdajs and associates analyzed 50 human hearts from cadavers without previous pathological alterations (Berdajs et al., 2003). They described the topographic relation between the sinus node artery and the superior posterior border of the interatrial septum with regard to the sinus node dysfunction that follows the superior septal approach to the mitral valve. The position of the sinus node and the course of the sinus node artery were investigated. For identification of the origin of the artery, selective coronary angiograms were performed. The course of the sinus node artery and its topographic relation to the interatrial septum was identified by the dry dissections of the hearts. Based on histologic and dry dissected specimens the exact position of the sinus node was determined. They found that the sinus node artery originates from the right coronary artery in $66 \%$ of examined cases and from the left coronary artery in 34\% of cases, and that the sinus node artery crosses the superior posterior border of the interatrial septum in $54 \%$ of cases. On the basis of morphological and clinical results, they concluded that the risk of intraoperative damage to the sinus node artery during the superior septal approach to the mitral valve is high.

Shin and associates assessed sinus node function after the superior septal approach in 46 patients (Sin et al., 2001). Twelve of 20 patients with preoperative sinus rhythm experienced early postoperative supraventricular arrhythmias, but all spontaneously recovered sinus 
rhythm. Electrophysiological studies revealed a lack of sinus node dysfunction. During the postoperative period ( $34+/-24$ months), 2 of the 20 patients with preoperative sinus rhythm developed persistent atrial fibrillation, and 3 of the 25 patients with preoperative atrial fibrillation achieved normal sinus srhythm. They concluded that the superior septal approach does not appear to cause longterm adverse effects on sinus node function, although temporary effects may occur.

\section{f. Electrical remodeling after mitral valve surgery:}

Berdais and associates retrospectively evaluated the causative mechanisms underlying postoperative atrioventricular block following mitral valve replacement and mitral valve annuloplasty (Berdajs et al., 2008). They analyzed 391 patients undergoing mitral valve replacement or ring annuloplasty and quadrangular resection between 1990 and 2003. Their exclusion criteria were preoperative atrioventricular block, two or three valvular procedures, reoperations and procedures combined with coronary artery bypass grafting. The presence of the postoperative atrioventricular block was compared with preoperative and intraoperative variables. On 55 post-mortem specimens the relationship between the atrioventricular node, atrioventricular node artery and mitral valve annulus was investigated. Patients' mean age was $59+/-14$ years and $44 \%$ of patients were female. Postoperatively atrioventricular block occurred in $92(23.5 \%)$ patients. Third degree atrioventricular block was found in $17(4 \%)$ patents, in whom a pacemaker was implanted within median interval of 4 days. Second degree atrioventricular block occurred and first degree atrioventricular block in five $(1.3 \%)$ and in $70(18 \%)$ patients respectively. In dry dissected human hearts in $23 \%$ of investigated cases the atrioventricular node artery was discovered to run close to the annulus of the mitral valve. Their morphological investigation showed that the atrioventricular node artery runs in close proximity to the annulus in $23 \%$ of cases. They speculated that damage of the atrioventricular node artery might play a role in development of atrioventricular block.

The PR intervals on electrocardiogram in our patients who had the superior septal approach were prolonged in the early postoperative phase but returned to normal range in the late phase. We introduce a new concept of electrical remodeling after cardiac surgery, which needs atrial or ventricular iincisions. Coronary artery revascularization can cause such a electrical remodeling because of postischemic reperfusion injuries.

\section{g. Newly developed collateral arteries and electrical remodeling:}

We hypothesize that newly developed collateral blood flow to the sinus node or an altered cardiac conduction system caused by the superior septal approach might contribute to transient conduction disturbances (Misawa, 2005). The numerous anastomoses between the surrounding atrial arteries and the arteriolar network of the sinus node, which were mentioned by Kovács (Kovács, 2003), would help lead to preferable clinical outcomes. Cardiac surgeons need to take into consideration the potential risk after the superior septal approach, as implied by Berdajs and colleagues. However, most arrhythmias are transient and can be controlled by ordinary perioperative measures.

The possible causes of the postoperative dysrhythmias with the superior septal approach are mentioned above. The prolongation of the PR interval in the superior septal approach patients in the early postoperative phase and return to normal in the late phase might be caused by newly developed collateral blood flows to the sinus node or cardiac conduction systems. Further studies are required to confirm these hypotheses. The superior septal approach group had the potential risk of an atrial septal defect, but postoperative echocardiography found no cases of such a defect. 
Conventional left atrial incision in the control group was longitudinal of approximately $5 \sim 6$ $\mathrm{cm}$. The sinus node artery is not sacrificed. Even in cases requiring tricuspid surgery, right atrial incision was shorter than the that in the superior septal approach group. This might result in the stable PR intervals after surgery.

\section{h. Postoperative rhythm management:}

In our study, patients with bradycardia below 70 beats/min were routinely paced during the early postoperative phase, and no patients developed medically intractable bradycardia more than 2 weeks postoperatively. In addition, no patient required implantation of a permanent pacemaker because of symptomatic or latent bradycardia as detected by Holter electrocardiograms at the median follow-up time of 15 months. As the extended superior septal approach, right atrial and septal incisions are joined at the superior end of the interatrial septum and extended across the dome of the left atrium to the base of the left atrial appendage. Our procedure includes a right atriotomy not going beyond the crista terminalis, an atrial septotomy beginning at the fossa ovalis, and a subsequent left atrial incision $2 \mathrm{~cm}$ long parallel to the superior vena cava. The limited incision may contribute to these minimization of postoperative conduction disturbances after the superior septal approach.

\section{i. A modified superior septal approach:}

Nguyen showed a modified version of the superior septal approach, which was made without cutting the right atrial appendage and the dome of the right atrium Nguyen, 2009). The modified approach includes three incisions. The first incision is made vertically on the anterolateral aspect of the right atrium, parallel to the atrioventricular groove, from the base of the inferior vena caval cannula to the root of the right atrial appendage. The second incision is made vertically on the transatrial septum, from the base of the inferior vena cava to the middle of the right atrial roof. The third incision extends the second incision $2-3 \mathrm{~cm}$ to the left atrial roof, and forward to the root of the left atrial appendage. The author evaluated this shorter procedure in 30 patients aged 4-61 years undergoing complex mitral valve operations including mitral repair $(33.3 \%)$, reoperation $(30 \%)$, and small left atrium $(30 \%)$. The myocardial ischemic time was $117+/-29.9$ minutes (range, 53-173). Cardiac rhythm resumed spontaneously after release of the aortic clamp in $93.3 \%$ of patients, including $36.7 \%$ who regained sinus rhythm from arrhythmia preoperatively. There was no heart block, bleeding, or mortality. The author insisted that the modified approach does not cut through the right atrial appendage and part of the right atrium, therefore, it can better nourish the sinus region from the collateral system around the sinus node, and increase the number of atrial muscle fibers that connect the sinus node with the atrioventricular node, thus reinforcing sinoatrial conduction.

\section{Conclusions}

Excellent visibility of the mitral valve is important for surgical treatment. It yields successful clinical results and educational advantages for surgical residents. Some clinical problems, including postoperative rhythm disturbances, can occur with the superior septal approach, but they can be controlled medically or with transient electrical pacemaking in the early postoperative phase. Conventional approaches for mitral surgery can also produce postoperative rhythm disturbances. We conclude that the superior septal approach is an excellent approach for mitral operations or left atrial tumor resection that overcomes postoperative transient dysrhythmias. 


\section{References}

Berdajs, D. Patonay,1. \& Turina, MI. (2003). The clinical anatomy of the sinus node artery. Ann Thorac Surg 76,3, (2003, September), 732-5, 0003-4975

Berdajs, D. Schurr, UP. Wagner, A. Seifert, B. Turina, MI. \& Genoni, M. (2008). Incidence and pathophysiology of atrioventricular block following mitral valve replacement and ring annuloplasty. Eur J Cardiothorac Surg 34,1, (2008, July), 55-61, 1010-7940

Edmunds, LH. Clark, RE. Cohn, LH. Grunkemeier, GL. Miller, DC. \& Weisel, RD. (1996). Guideline for reporting morbidity and mortality aftercardiac valvular operations. Ann Thorac Surg 62,3,(1996, September)932-935, 0003-4975

Emkanjoo, Z. Mirza-Ali, M. Alizadeh, A. Hosseini,S. Jorat, MV. Nikoo, MH. \& Sadr-Ameli, MA. (2008). Predictors and frequency of conduction disturbances after open-heart surgery. Indian Pacing Electrophysiol J 8,1, (2008, February), 14-21, 0972-6292

Gaudino, M. Alessandrini, F. Glieca, F. Martinelli, L. Santarelli, P. Bruno, P. \& Possati, G. (1997). Conventional left atrial versus superior septal approach for mitral valve replacement. Ann Thorac Surg 63,4,(1997, April),1123-7, 0003-4975

Goldman, BS. Hill, TJ. Weisel, RD. Scully, HE. Mickleborough, LL. Pym, J. \& Baird, RJ. Permanent cardiac pacing after open-heart surgery: acquired heart disease. Pacing Clin Electrophysio 7,3, (1984, May), 367-71, 0147-8389

Kirklin, JW. \& Barratt-Boyes, BG. (1993). Cardiac Surgery. 2nd ed. Churchill Livingstone, 0443-07526-3, New York

Kon, ND. Tucker, WY. Mills, SA. Lavender, SW. \& Cordell, AR. (1993). Mitral valve operation via an extended transseptal approach. Ann Thorac Surg 55,6,(1993, June), 1413-7, 0003-4975

Kovács, GS. (2003). The clinical anatomy of the sinus node artery, Ann Thorac Surg 76,3, (2003, September), 735-736, 0003-4975

Lukac, P. Hjortda, VE. Pedersen, AK. Mortensen, PT. Jensen, HK. \& Hansen, PS. (2007). Superior transseptal approach to mitral valve is associated with a higher need for pacemaker implantation than the left atrial approach. Ann Thorac Surg 83,1,(2007, January), 77-82, 0003-4975

Masuda, M. Tominaga, R. Kawachi, Y. Fukumura, F. Morita, S. Imoto, Y. Toshima, Y. Tomita, Y. \& Yasui, H. (1996). Postoperative cardiac rhythms with superior septal approach and lateral approach to the mitral valve. Ann Thorac Surg 62,4, (1996, October), 1118-22, 0003-4975

Merin, O. Ilan, M. Oren, A. Fink, D. Deeb, M. Bitran, D. \& Silberman, S. (2009). Permanent pacemaker implantation following cardiac surgery: indications and long-term follow-up. Pacing Clin Electrophysiol 32,1, (2009, January), 7-12, 0147-8389

Misawa, Y. Fuse, K. Kawahito, K. Saito, T. \& Konishi, H. (1999). Conduction disturbances after superior septal approach for mitral valve repair. Ann Thorac Surg 68, 4, (1999, October), 1262-5, 0003-4975

Misawa, Y. Saito, T. Fuse, K. Sohara, Y. (2002). Endoscope-assisted superior septal approach for resection of left atrial myxoma. J Thorac Cardiovasc Surg 123, 2, (2002, February), 357-358, 0022-5223

Misawa, Y. \& Kaminishi, Y.(2004). Early and late arrhythmias in patients in preoperative sinus rhythm after superior septal approach. Ann Thorac Surg 77, 6, (2004, June), 2259-9, 0003-4975 
Misawa, Y. Kaminishi, Y. Sakano, Y. (2005). Conduction disturbance after shutdown of the sinus node artery. Ann Thorac Surg 79, 1, (2005, January), 388-9, 0003-4975

Nguyan, HU (2009). Improved combied superior-transseptal approach to the mitral valve. Asian Cardiovasc Thorac Ann 17,2(2009, April), 171-4, 0218-4923

Silva Junior, JR. Ferreira, CA. Rodrigues, AJ. Vicente, WV. \& Evora PR. (2008). Sinus node function in patients operated for mitral valve disease; Indirect evaluation with epimyocardial electrodes. Acta Cir Bras 23, Suppl 1, (2008), 126-32, 0102-8650

Shin, H. Yozu, R. Higashi, S.Kawada, S. (2001). Sinus node function after mitral valve surgery using the suoerior septal approach. Ann Thorac Surg 71, 2, (2001, February), 587-90, 0003-4975

Smith, CR. (1992). Septal-superior exposure of the mitral valve. The transplant approach. J Thorac Cardiovasc Surg 103, 4, (1992, April), 623-8, 0022-5223

Takeshita, M. Furuse, A. Kotsuka, Y. \& Kubota, H. (1997). Sinus node function after mitral valve surgery via the transseptal superior approach. Eur J Cardiothorac Surg 12, 3, (1997, September), 341-4, 1010-7940

Utley, JR. Leyland, SA. \& Nguyenduy T. (1995). Comparison of outcomes with three atrial incision for mitral valve operations. J Thorac Cardiovasc Surg 109,3, (1995, March), $582-7,0022-5223$

Yamada, T. McElderry, HAT. Doppalapudi, H. Epstein, AE. Plumb, VJ. \& Kay. GN. (2009). Catheter ablation of premature ventricular contractions arising from the mitral annulus after mitral valvoplasty. Pacing Clin Electrophysiol 32,6, (2009, June), 825-7, 0147-8389 


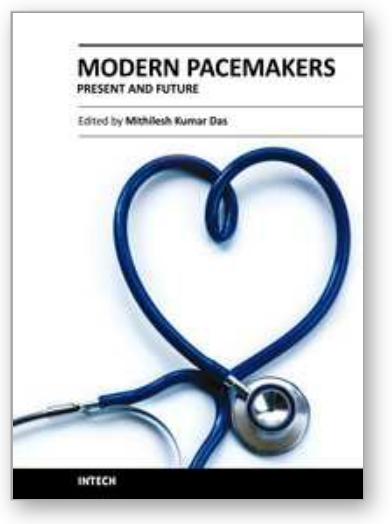

\author{
Modern Pacemakers - Present and Future \\ Edited by Prof. Mithilesh R Das
}

ISBN 978-953-307-214-2

Hard cover, 610 pages

Publisher InTech

Published online 14, February, 2011

Published in print edition February, 2011

The book focuses upon clinical as well as engineering aspects of modern cardiac pacemakers. Modern pacemaker functions, implant techniques, various complications related to implant and complications during follow-up are covered. The issue of interaction between magnetic resonance imaging and pacemakers are well discussed. Chapters are also included discussing the role of pacemakers in congenital and acquired conduction disease. Apart from pacing for bradycardia, the role of pacemakers in cardiac resynchronization therapy has been an important aspect of management of advanced heart failure. The book provides an excellent overview of implantation techniques as well as benefits and limitations of cardiac resynchronization therapy. Pacemaker follow-up with remote monitoring is getting more and more acceptance in clinical practice; therefore, chapters related to various aspects of remote monitoring are also incorporated in the book. The current aspect of cardiac pacemaker physiology and role of cardiac ion channels, as well as the present and future of biopacemakers are included to glimpse into the future management of conductions system diseases. We have also included chapters regarding gut pacemakers as well as pacemaker mechanisms of neural networks. Therefore, the book covers the entire spectrum of modern pacemaker therapy including implant techniques, device related complications, interactions, limitations, and benefits (including the role of pacing role in heart failure), as well as future prospects of cardiac pacing.

\title{
How to reference
}

In order to correctly reference this scholarly work, feel free to copy and paste the following:

Yoshio Misawa, Shin-ichi Ohki, Yuichiro Kaminishi, Yasuhito Sakano, Kei Aizawa, Tsutomu Saito, Hiroaki Konishi and Arata Muraoka (2011). Conduction Disturbances after the Superior Septal Approach for Mitral Valve Repair, Modern Pacemakers - Present and Future, Prof. Mithilesh R Das (Ed.), ISBN: 978-953-307-2142, InTech, Available from: http://www.intechopen.com/books/modern-pacemakers-present-andfuture/conduction-disturbances-after-the-superior-septal-approach-for-mitral-valve-repair

\section{INTECH}

open science | open minds

\section{InTech Europe}

University Campus STeP Ri

Slavka Krautzeka 83/A

51000 Rijeka, Croatia

Phone: +385 (51) 770447

\section{InTech China}

Unit 405, Office Block, Hotel Equatorial Shanghai

No.65, Yan An Road (West), Shanghai, 200040, China

中国上海市延安西路65号上海国际贵都大饭店办公楼 405 单元

Phone: +86-21-62489820 
Fax: +385 (51) 686166

Fax: +86-21-62489821

www.intechopen.com 
(C) 2011 The Author(s). Licensee IntechOpen. This chapter is distributed under the terms of the Creative Commons Attribution-NonCommercialShareAlike-3.0 License, which permits use, distribution and reproduction for non-commercial purposes, provided the original is properly cited and derivative works building on this content are distributed under the same license. 